\title{
Representaciones sociales de la violencia en jóvenes ecuatorianos
}

\author{
Marisol Elizalde Monjardin ${ }^{1}$, Hiram Reyes-Sosa ${ }^{2}$ \\ Universidad Autónoma de Sinaloa, México
}

Los estudios de la violencia en Ecuador se han caracterizado por su teorización y medición mediante frecuencias delictivas sin tomar en cuenta su carácter subjetivo. El presente estudio tuvo como objetivo conocer la representación social que los jóvenes ecuatorianos tienen de la violencia. Se aplicó un cuestionario abierto para conocer sus contenidos y significados. Una muestra de 298 jóvenes universitarios de la ciudad de Ambato, con una media de edad de 21.28 años $(D T=1.61)$, participaron en esta investigación. Los resultados muestran que los jóvenes perciben un clima de inseguridad, caracterizados por delitos comunes (asaltos y robos) y fallas en la estructura del sistema gubernamental (policial). Así, se ha establecido un clima colectivo caracterizado por la inseguridad y la incertidumbre.

Palabras clave: Representaciones sociales, procesual, violencia, inseguridad, Ecuador.

\section{Social representation of violence in young Ecuadorians}

Studies of violence in Ecuador have been characterized by theorization and measurement of criminal frequency, without taking into account subjective dimensions. The present study aimed to explore the social representation that young Ecuadorians have of violence. Thus, an open questionnaire was administered to understand the content and meaning of violence. A sample of 298 university students from Ambato city, with a mean age of 21.28 years $(S D=1.61)$ participated in this study. The results show that the participants perceive a climate of insecurity, defined by common crime (muggings and robbery) and failures in the structure of the governmental system (police). Consequently, a collective climate defined by fear and uncertainty exists.

Key words: Social representations, procedural, violence, insecurity, Ecuador.

\section{Representaçóes sociais de violência em jovens equatorianos}

Os estudos da violência no Equador vêm se caracterizando por sua teorização e medição mediante frequências delitivas, sem levar em conta seu caráter subjetivo. O presente estudo teve como objetivo conhecer a representaçáo social que os jovens equatorianos têm da

1 Doctora en Investigación Psicológica por la University of Leicester. Profesora en la Facultad de Psicología de la Universidad Autónoma de Sinaloa. Dirección Postal: Ciudad Universitaria, Av. De las Américas y Blvd. Universitarios s/n, Culiacán, Sinaloa, México, CP. 80080. Contacto: melizalde@uas.edu.mx ORCID: https://orcid.org/0000-0001-5625-7233

2 Doctor en Psicología Social y Metodología de las Ciencias del Comportamiento por la Universidad del País Vasco. Profesor en la Facultad de Psicología de la Universidad Autónoma de Sinaloa. Dirección Postal: Ciudad Universitaria, Av. De las Américas y Blvd. Universitarios s/n, Culiacán, Sinaloa, México, CP. 80080. Contacto: hiramreyez@gmail.com ORCID: https://orcid.org/0000-0001- 6763-847X 
violência. Aplicou-se um questionário aberto para conhecer seus conteúdos e significados. Uma amostra de 298 jovens universitários da cidade de Ambato, com uma média de idade de 21,28 anos $(D P=1.61)$, participaram desta pesquisa. Os resultados mostram que os jovens percebem um clima de insegurança, caracterizados por delitos comuns (assaltos e roubos) e falhas na estrutura do sistema governamental (policial). Assim, estabeleceu-se um clima coletivo caracterizado pela insegurança e incerteza.

Palavras-chave: Representaçóes sociais, processual, violência, insegurança, Equador.

\section{Représentations sociales de la violence des jeunes équatoriens}

Les études concernant la violence en Equateur se caractérisent $d$ une part par sa théorisation et $\mathrm{d}$ autre part par la mesure des délits criminels, ce qui met de côté toute subjectivité. Cette étude a comme objectif de connaître la représentation sociale de la violence par les jeunes équatoriens. Un questionnaire ouvert a été appliqué pour connaître son contenu et son sens. Un groupe de 298 jeunes étudiants de la ville de Ambato, avec comme moyenne d âge 21.28 ans $(D T=1.61)$, ont participé à cette étude. Les résultats montrent que les jeunes perçoivent un climat $\mathrm{d}$ insécurité caractérisé par : des délits communs (vols et braquages) et des failles dans la structure du système gouvernemental (policier). Ainsi s est instauré un climat collectif $\mathrm{d}$ insécurité et $\mathrm{d}$ incertitudes.

Mots clés: Représentations sociales, processuel, violencia, insécurité, Equateur. 
La violencia ha sido un fenómeno que históricamente ha causado fuertes estragos en la sociedad ecuatoriana (Palomeque, 2002). Uno de sus efectos más visibles es la inseguridad, que a partir de los años noventa a la actualidad, ha presentado un aumento significativo (Carrión, 2003; Molina-Coloma, Reyes-Sosa \& Larrañaga-Egilegor, 2015; Núnez, 2011). A partir de las estadísticas del Instituto Nacional de Estadística y Censos (INEC) (2011), se puede observar que los delitos con mayor presencia en Ecuador son los robos a personas (13.6\%), los homicidios (19\%) y los secuestros (41\%). Además, en lo que corresponde a la percepción de inseguridad, se encontró que el $83.1 \%$ de la población señala que su ciudad es insegura y el $77.3 \%$ que la inseguridad es un problema que va en aumento (INEC, 2011). A pesar de que los datos estadísticos citados se aproximan a describir una violencia de tipo cotidiana (Molina-Coloma et al., 2015), se puede encontrar que los estudios que se han realizado dentro de Ecuador sobre la violencia, no han tomado en cuenta el contexto mediato (ver Ojeda, 2009; Palomeque, 2002; Torres, 2005). Concretamente, los estudios sobre dicho fenómeno se han desarrollado en tres sentidos: uno desde una visión teórica, otro desde las bases de datos institucionales (judiciales) y otro desde la seguridad ciudadana (Pontón, 2004).

En lo que concierne a la visión teórica, los trabajos realizados en Ecuador se han basado en estudios externos y han intentado argumentar a qué o cómo se puede categorizar la violencia (Molina-Coloma, Pérez \& Salaberría, 2018; Palomeque, 2002; Torres, 2005). Se ha sugerido que la violencia es una acción intencional (fuerza o poder) que produce o se categoriza como física, psicológica o sexual (Concha-Eastman, 2002). Desde las bases de datos institucionales gubernamentales y no gubernamentales (Centro de Estudios y Datos [CEDATOS], 2011; INEC, 2011), el estudio de este fenómeno se ha centrado en medir solo la frecuencia delictiva. De esta manera, solo se han cuantificado los 
hechos violentos que los propios ciudadanos denuncian ante un delito (INEC, 2011; Torres, 2005). Retomando los datos del propio INEC (2011), se ha encontrado que el 38.5\% de la población no denuncia algún tipo de delito por desconfianza al sistema judicial y particularmente desconfianza a la policía. Por lo tanto, puede existir un sesgo en los tipos de delito que en mayor medida se reportan, debido a que no necesariamente concuerdan con los delitos que se cometieron (cifra negra). Estas perspectivas de la violencia, si bien aportan datos relevantes sobre los hechos y acciones violentas que tienen mayor presencia entre la sociedad, también es verdad que permiten cuestionar la adecuación de los discursos que las instituciones gubernamentales han establecido en Ecuador sobre la violencia; tal y como se ha seńalado, estas visiones no han considerado el contexto ni quien padece la violencia en su vida diaria que sería el sujeto cotidiano (Paéz, 2004; Pontón, 2004).

La tercera visión de la violencia que se considera pertinente destacar y que es relevante para este estudio, es la que ha emergido conjuntamente en base del concepto de seguridad ciudadana (Carrión, 2003; Palomeque, 2002). Dicho concepto hace referencia a los derechos que tienen las personas a vivir una vida de libertad y dignidad; es decir, los derechos universales, inalienables e indivisibles que poseen las víctimas actuales y potenciales de la delincuencia (Informe sobre Desarrollo Humano para América Central [IDHAC], 2010). Desde la perspectiva de la seguridad ciudadana, se ha propuesto que la violencia tiene una causalidad en tres dimensiones: la política, económica y social. Así, se puede argumentar que la violencia puede emerger por factores estructurales (desigualdad o ingobernabilidad), institucionales (impunidad o colusión), y situacionales (delito o porte de armas) (Palomeque, 2002). Esta visión de la violencia permite plantear una aproximación cualitativa que considera a los sujetos sociales y permite comprender qué es la violencia y cómo afecta la vida cotidiana de la sociedad (San Martín, 2013).

El objetivo del presente estudio es conocer la representación social que los jóvenes ecuatorianos tienen de la violencia. Para el objetivo citado, se retomó la Teoría de las Representaciones Sociales (TRS), 
mediante la TRS se puede acceder a los discursos que la sociedad tiene sobre un objeto de relevancia social (Moscovici y Duveen, 2000). Por último, cabe señalar que se ha optado trabajar con el grupo de jóvenes, dado que, según la Primera Encuesta Nacional sobre Jóvenes y Participación Política en Ecuador (Ramírez, 2011), se ha encontrado que son los jóvenes los más afectado por problemáticas como la inseguridad (10.3\%) y la delincuencia (52\%). De igual manera, y según los datos de INEC (2011) de la población ecuatoriana, es el grupo de los jóvenes (42.7\%) entre 16 y 27 años quienes en mayor medida han sido víctimas de algún tipo de delito.

\section{Representaciones sociales: una visión del pensamiento social}

Las Representaciones Sociales (en adelante RS) se pueden entender como modos de reconstrucción de la realidad material o simbólica existentes, más que construcciones de la realidad social (Cerrato \& Palmonari, 2007; Gaffié, 2012). Por ello, desde la perspectiva de las RS más que conocer las imágenes cosificadas de la realidad (prácticas o representaciones establecidas frente a objetos), se busca conocer cómo es que ciertos objetos, al entrar al campo de interés social (sujeto dialógico), reconstruyen las prácticas y las formas de pensamiento cotidiano (Markova, 2003; Rodríguez, 2003).

Desde la perspectiva de Moscovici (2001), las RS pueden ser entendidas como una lógica de pensamiento constituido (contenido). Por tanto, las RS son una especie de producto que se encuentra en la mente de los individuos, afectado siempre por el contexto mediato en el que se encuentran los sujetos (Valencia, Gil-De-Montes \& Elejabarrieta; 2004). En este sentido, las RS son concebidas como una noción cognitiva que al mismo tiempo se instala y obedece a un nivel social; es decir, las imágenes que los sujetos reconstruyen son siempre afectadas por la estructura social (Farah, 2011; Sarrica \& Contarello, 2004). A pesar de que las RS pueden ser analizadas también como un proceso, para fines de esta investigación se tiene particular interés por el contenido (producto). Identificar el contenido de RS permitirá conocer los productos socioculturales que los grupos, en un determinado contexto, 
construyen frente a un objeto de interés social (Cerrato \& Palmonari, 2007; Doise, 2003; Wagner, Hayes \& Flores, 2011).

Con la finalidad de identificar los contenidos de un objeto (la violencia) de representación, es necesario profundizar en las tres dimensiones que construyen a una RS (Moscovici \& Duveen, 2000; Wagner et al., 2011). La dimensión de información comprende el conjunto de conocimientos que un grupo posee sobre un objeto de interés social; es decir, la calidad y la cantidad de información que un grupo tiene sobre el fenómeno de interés. La dimensión del campo representacional se relaciona con el contenido concreto y estructurado de las informaciones que un grupo construye sobre el objeto de representación; en concreto, es el modo de clasificar la información con el objetivo de establecer relaciones y comparaciones entre los distintos elementos de la representación. La dimensión de la actitud se relaciona con la posición evaluativa (positiva o negativa) que un grupo tiene frente al objeto de representación social; esta dimensión es más funcional, ya que tiene estrecha relación con la proximidad que el grupo mantiene con el objeto de representación (Moscovici, 2001; San Martín, 2013). Desde la perspectiva de Cerrato y Palmonari (2007), al realizar una comparación entre las dimensiones de la RS se puede comprender de mejor manera los contenidos y significados que los grupos tienen del objeto de estudio, este caso de la violencia.

Partiendo de la revisión de literatura se pretende conocer la RS de la violencia en jóvenes ecuatorianos. Para ello, en un primer momento, se busca realizar una clasificación jerárquica descendente que permite identificar los distintos discursos que los jóvenes emplean para definir la violencia. En un segundo momento, se podrá conocer la distancia entre los distintos discursos empleados por los jóvenes al representar la violencia a través de un análisis de correspondencia. Finalmente, se realizará un análisis de similitud que permite conocer la manera en que se relacionan los discursos que los jóvenes tienen sobre la violencia. 


\section{Método}

\section{Participantes}

Un estudio transversal con una muestra no probabilística intencional en el cual 298 jóvenes universitarios participaron en esta investigación. Los jóvenes pertenecían a tres carreras de la Universidad Técnica de Ambato (UTA), en Ecuador: Licenciatura en Psicología, Licenciatura en Informática y Licenciatura en Administración. De los participantes un $47.7 \%$ eran hombres y un $52.3 \%$ eran mujeres. La media de edad de los jóvenes era de $21.28(D T=1.62)$.

\section{Medición}

Con la finalidad de conocer los contenidos y los significados de la RS de la violencia, se optó por un cuestionario abierto. Desde la perspectiva de Moscovici y Duveen (2000), los cuestionarios permiten acceder a los elementos de la RS. En la primera sección, se solicitaba a cada participante que enunciase los elementos que consideraban necesarios para explicar las causas de la violencia. La pregunta inductora fue la siguiente ¿A qué causas, prácticas o factores culturales considera usted que la sociedad ecuatoriana ha atribuido y, por consecuencia, ha agudizado el fenómeno de la violencia? En la segunda sección, se solicitaba que justificasen el porqué de cada evocación asociada a las causas de la violencia. Finalmente, en la tercera sección se solicitaba que jerarquizaran las tres respuestas en el orden de importancia que para ellos tenía cada evocación. Para el presente estudio, únicamente la segunda sección que explicaba las causas de la violencia fue analizada, ya que esta refleja los contenidos de la RS.

\section{Procedimiento}

Con el propósito de realizar la aplicación de los cuestionarios, se realizó una presentación de los objetivos en primer lugar a directivos y en segunda instancia a los jóvenes participantes. Una vez obtenido el permiso de los directivos, de manera colectiva se abordó a los jóvenes. 
Previamente firmaron un formato de consentimiento, que declaraba que todas las respuestas eran completamente anónimas. Posteriormente respondieron de manera individual y voluntaria el cuestionario.

\section{Análisis de datos}

El análisis de los datos, se realizó mediante el software IRaMuTeQ 7 (Interface de R pour les Analyses Multidimensionneles de Textos et de Questionnaires). Este es un software libre que permite realizar análisis multidimensionales de textos de diferente naturaleza por ejemplo, entrevistas o cuestionarios de preguntas abiertas. Mediante IRaMuTeQ 7 , se pueden encontrar patrones discursivos (mundos lexicales) puesto que en sus análisis, realiza comparaciones entre los perfiles-columnas y los perfiles-filas (Ratinaud \& Déjean, 2009). IRaMuTeQ 7 antes de lanzar el análisis, lematiza las palabras para transformarlas a su forma canónica o su raíz (Carneiro, Tsunoda \& Giacomiti, 2017). A través del estadístico chi cuadrado, se obtienen los índices de distancia que permite evidenciar el grado de proximidad o disparidad entre las categorías construidas (Garnier \& Guérin, 2010).

El software IRaMuTeQ 7 realiza diferentes tipos de análisis, por ejemplo, de comparaciones grupales, de clasificación jerárquica descendiente, de similitud y de nubes de palabras. Para esta investigación se ha optado por utilizar el análisis de clasificación jerárquica descendiente (algoritmo de cluster) que permite obtener patrones y segmentos (categorías) discursivos. Por otra parte, con la finalidad de conocer y representar en un espacio las categorías creadas, se utilizó el análisis de correspondencia; este análisis permite representar los discursos en un espacio gráfico dando sentido a los discursos construidos (Greenacre, 2010). Por último, con el objetivo de observar la distribución y la conexión entre los diferentes discursos, se utilizó el análisis de similitud. Dicho análisis permite demostrar las relaciones entre los elementos de cada categoría construida. 


\section{Resultados}

\section{Características del cuerpo de palabras}

El cuerpo de datos está constituido por 1815 palabras. Las Unidades Iniciales de Contexto (UIC) fueron en un principio analizadas por el programa IRaMuTeQ 7. Cabe señalar que los índices arrojados por IRaMuTeQ 7 demostraron que el contenido es aceptable para realizar los análisis posteriores (62.89\% HAPAX 36\%; type/token ratio $30 \%)$ (ver Tabla 1).

\section{Tabla 1}

Características del cuerpo

Número de textos 266

Número de ocurrencias 1815

Número de formas $\quad 539$

Número de hápax $\quad 339$ (62.89\% de formas - $18.68 \%$ de ocurrencias)

El cuerpo de datos fue sometido a un proceso de lematización basado en el diccionario en español. Dicho proceso se realiza para agrupar las palabras singulares, plurales y verbos conjugados a una misma forma singular basados en la raíz de la palabra o morfemas lexicales. El objetivo de este proceso es tratar las diferentes palabras como sinónimos, para evitar que se analicen como unidades separadas. $\mathrm{Al}$ concluir el proceso de lematización se encontró un decremento en el número de formas de HAPAX a 58.15\% y en el type/token ratio 25\%, lo cual hizo que el análisis del texto fuese más estable (ver Figura 1).

\section{Clasificación jerárquica descendiente}

A continuación, se realizó un proceso conocido como "Parsing" de las UCE (Unidades de Contexto Elementales). Dicho proceso se refiere a la división en partes del cuerpo de palabras y una posterior reagrupación, de esta manera, se pretende obtener un orden que permita una 
comprensión clara del texto; las agrupaciones creadas son conocidas como clusters.

El programa de IRaMuTeQ 7, crea clusters de acuerdo al vocabulario encontrado en la categorización de las palabras del texto. Estos clusters quedan representados en un dendograma, el cual después de agrupar todas las palabras, les da un orden jerárquico descendente (ver Figura 2). El orden descendente ayuda a maximizar la similitud entre las palabras del cluster, a su vez maximiza las diferencias entre los clusters. Dicho orden está dictaminado por el coeficiente de chi cuadrada. Las palabras que aparecen en primer lugar son las que contribuyen más a la organización del cluster, mientras que las últimas contribuyen menos. Como se aprecia en la Figura 2, las palabras de cada cluster se distinguen por un color específico.

Para el presente cuerpo de palabras, se aprecian cuatro clusters. El cluster 1, representa el 27.2\% de las UCE del cuerpo de palabras. Este cluster, está caracterizado por palabras como robo $\left(\chi^{2}=116.14\right.$; $p=.001)$, asalto $\left(\chi^{2}=46.93 ; p=.001\right)$, violencia $\left(\chi^{2}=23.87 ; p=.001\right)$, maltrato $\left(\chi^{2}=20.29 ; p=.001\right)$ y muerte $\left(\chi^{2}=19.41 ; p=.001\right)$. Como se puede apreciar, este conjunto palabras hace referencia a la información general que los jóvenes tienen sobre la violencia. Desde el discurso de los jóvenes, se puede observar diferentes delitos (robos, asaltos o secuestros) que padecen en su vida cotidiana; además, de los diferentes contextos en donde se presentan dichos delitos (buses o calles). El cluster 2, concentra el $20.4 \%$ de las UCE del cuerpo de palabras. Las palabras que encabezan este cluster son aquellas como peligro $\left(\chi^{2}=45.28 ; p=.001\right)$, inestabilidad $\left(\chi^{2}=23.01 ; p=.001\right)$, sentir $\left(\chi^{2}=15.93 ; p=.001\right)$, pasar $\left(\chi^{2}=15.93 ; p=.001\right)$ y daño $\left(\chi^{2}=11.63 ; p=.001\right)$. De esta forma, se puede concluir que este cluster hace referencia a los significados que los jóvenes han construido en su vida cotidiana frente a la violencia; los jóvenes han desarrollado prácticas (actitudes) de temor en sus interacciones sociales ya que han desarrollado un fuerte sentimiento de inseguridad frente al contexto. 
Representaciones sociales de la violencia en jóvenes ecuatorianos / Elizalde Monjardin, Reyes-Sosa

\section{Resumen}

Número de textos: 266

Número de ocurrencias: 1815

Número de formas: 454

Número de hápax: 264 (14.55\% de ocurrencias - $58.15 \%$ de formas)

Media de ocurrencias por texto: 6.82

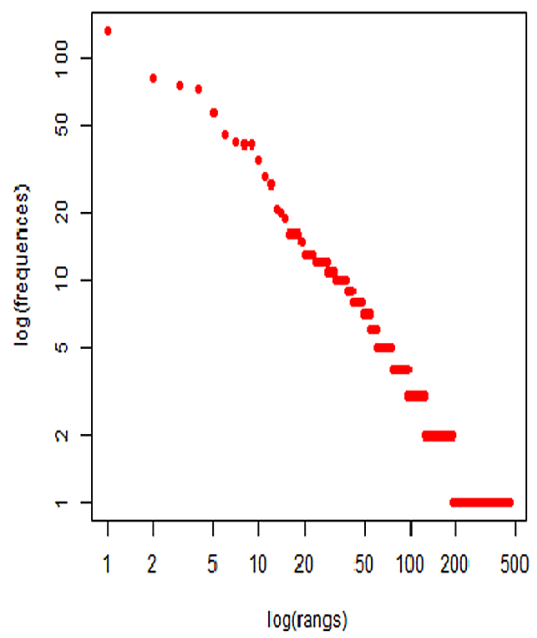

Figura 1. Características del cuerpo después de la legitimación

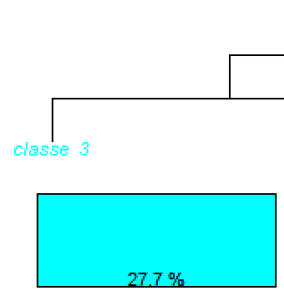

$277 \%$
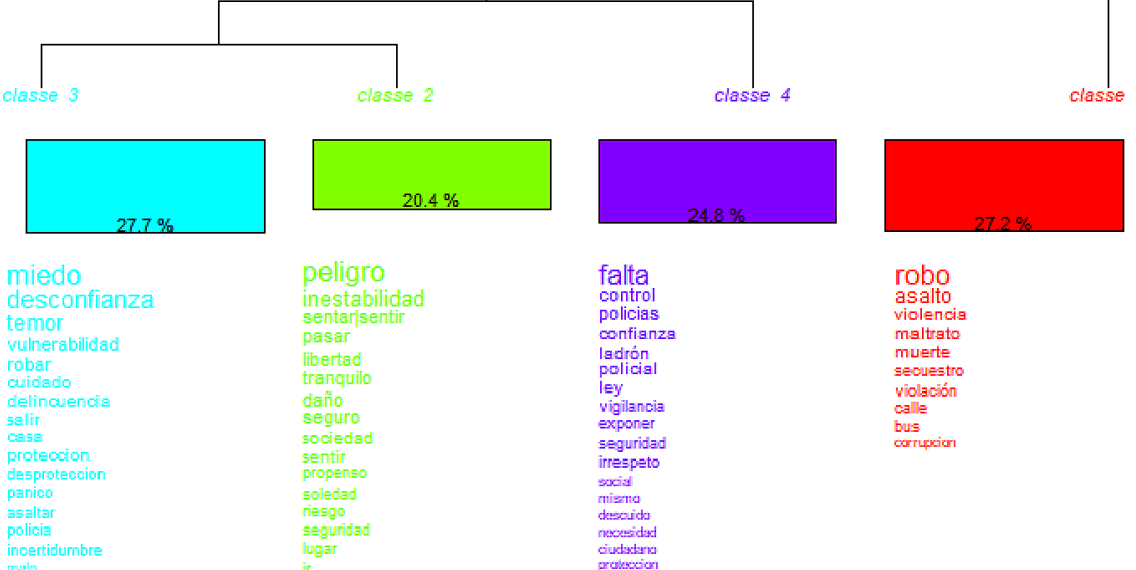

inestabilidad

control

pasar

libertad.

tranquilo

danio

seguro

sociedad

propenso

soledad.

riesgo

geguridad

robo

asalto

violencia

confianza

ladrón

policial

ley

maltrato

muerte

secuestro

vigilancia

exponer

seguridad

yidacio

cale

bue
ournupion

irrespeto

social

misma

disouide

enseseidyd

ciudsaderias

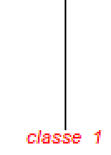

Figura 2. Dendograma de clusters 
El cluster 3 concentra el $27.7 \%$ de las UCE del cuerpo de palabras. Las palabras que definen este cluster se refieren a los sentimientos personales como el miedo $\left(\chi^{2}=49.99 ; p=.001\right)$, la desconfianza $\left(\chi^{2}=36.38 ; p=.001\right)$, el temor $\left(\chi^{2}=26.08 ; p=.001\right)$ y la vulnerabilidad $\left(\chi^{2}=14.38 ; p=.001\right)$. En su contenido, este cluster destaca las emociones negativas (incertidumbre) suscitadas por el hecho de transitar o salir por las calles. Dicho de otro modo, los jóvenes tienen presentes las emociones de miedo por la inestabilidad que presenta su contexto cotidiano. El cluster 4 está compuesto por el $24.8 \%$ de las UCE del cuerpo. El tono general de este cluster está definido por palabras como la falta $\left(\chi^{2}=107.27 ; p=.001\right)$, control $\left(\chi^{2}=30.62 ; p=.001\right)$, policías $\left(\chi^{2}=28.06 ; p=.001\right)$ y confianza $\left(\chi^{2}=25.03 ; p=.001\right)$. Por consiguiente, desde la perspectiva de los jóvenes, si la violencia ha presentado un aumento, es debido a la falta de una estructura jurídica institucional. Por tal razón, dicho cluster se caracteriza por destacar en su contenido, la falta de control institucional (leyes) relacionada con los policías o la ausencia de vigilancia.

\section{Análisis de correspondencia}

El programa IRaMuTeQ 7 arroja una distribución en cuadrantes. Dicha distribución es generada a partir de una tabla de contingencia y permite observar gráficamente la proximidad de las palabras de cada cluster (ver Figura 3). Para ello, IRaMuTeQ7 crea una división entre los 4 clusters, identificando cada uno con su color específico pre-asignado. Dentro de cada sección se encuentran las palabras más representativas del grupo, las que contribuyen en mayor medida a la conformación del cluster se encuentran en fuente de mayor tamaño, disminuyendo de tamaño en relación con su contribución al cluster. 


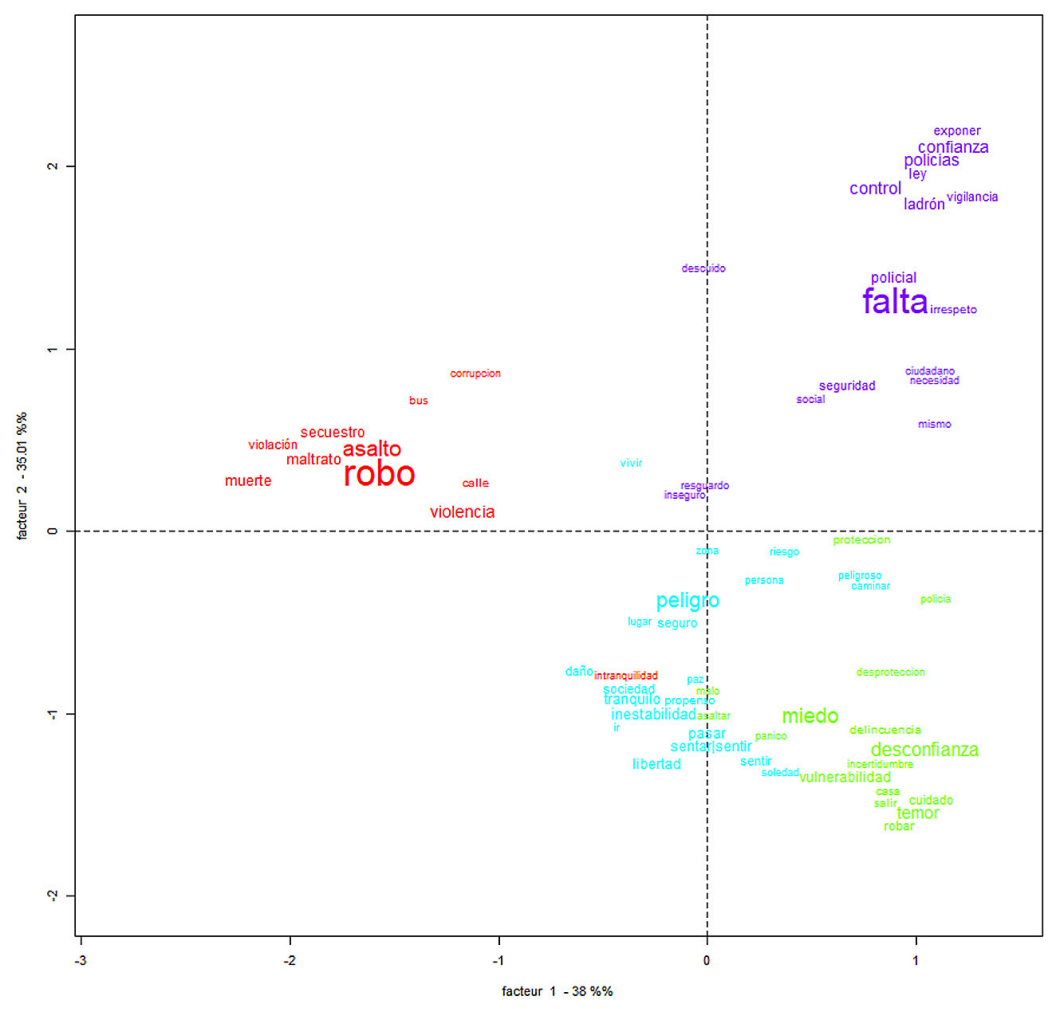

Figura 3. Distribución en cuadrantes ilustrando las variaciones de contingencia

Como se puede apreciar en la figura 3, existen tres discursos diferenciados: el primero, ubicado en la parte superior izquierda, el segundo ubicado en la parte inferior derecha y el tercero ubicado en la parte superior derecha. La distancia entre ellos, posibilita la diferenciación de los tres discursos, lo cual a su vez permite identificar a qué dimensión de la representación pertenece cada uno (Cerrato \& Palmonari, 2007). En este sentido, en base al análisis de cluster y de correspondencia, se puede determinar que el cluster 2 y 3 hacen referencia a la dimensión actitudinal de la representación. En su contenido, este cluster enfatiza los 
sentimientos de incertidumbre y miedo que los jóvenes han reconstruido frente a su medio social, en el cual destacan las emociones negativas provocadas por el hecho de transitar o salir a la calle. El segundo discurso, hace referencia a las causas que han permitido el aumento de la violencia, y se encuentra representado en el cluster 4. De esta manera, los jóvenes destacan la ineficiente estructura gubernamental (falta de estructura judicial) como un problema que ha provocado el aumento de los hechos violentos en la vida cotidiana. Es decir, en este discurso se destacan los contenidos concretos y estructurados que permiten explicar las causas por las cuales un fenómeno como la violencia puede establecerse y aumentar en un contexto determinado, lo que categoriza concretamente la dimensión del campo representacional de la representación. Por último, y como tercer discurso, se puede observar que el cluster 1 hace referencia a las consecuencias que tiene la violencia en la cotidianidad. Los jóvenes destacan los diferentes hechos violentos que padecen en su vida diaria, por ejemplo, los robos y los asaltos en autobuses o en la vía pública. Este cluster destaca la información (consecuencias) concreta que los jóvenes tienen de la violencia en su contexto mediato, lo que permite evidenciar que el cluster 1 se ubica en la dimensión informativa del campo representacional.

\section{Análisis de similitud}

Para concluir con el análisis de los clusters, se presenta una distribución gráfica en forma de ramificaciones de las palabras que encabezan cada cluster y que constituyen el mismo (ver Figura 4). Como se mencionó anteriormente, las palabras con mayor impacto en el cluster se encuentran en fuente de mayor tamaño. Se puede apreciar de manera clara la palabra robo la cual encabeza el cluster 1, con una ocurrencia de 81 veces, siendo la que con más frecuencia se encontró en el cuerpo de palabras. Esta se encuentra seguida por las palabras miedo (encabezando el cluster 3) y falta (encabezando el cluster 4) con una ocurrencia de 75 y 72 veces respectivamente. Encabezando el cluster 2, se encuentra la palabra peligro, con una ocurrencia de 35 , la cual es precedida por violencia con una ocurrencia de 41, pero del cluster 1 . 


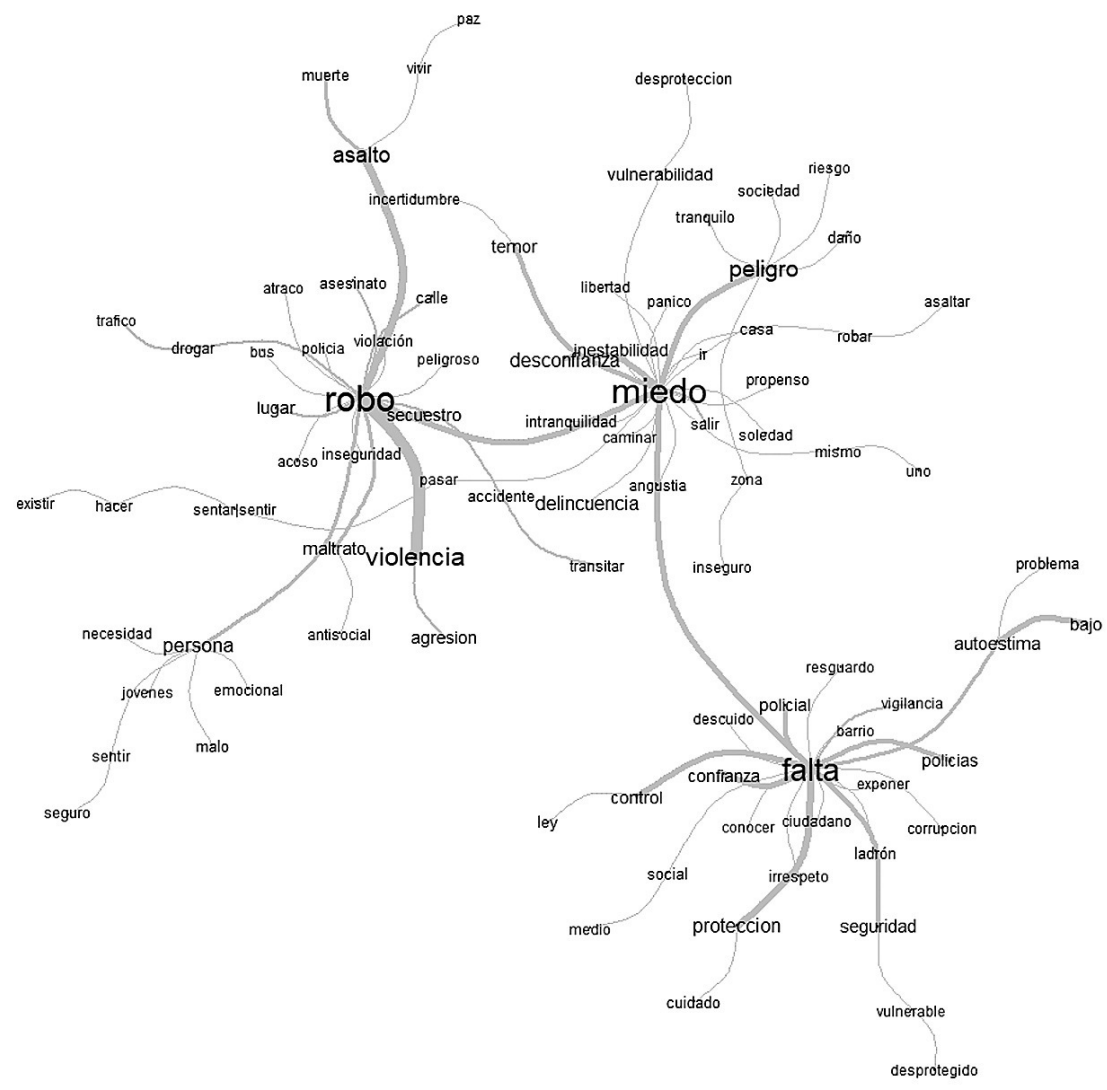

Figura 4. Gráfica de ramificación de palabras

La bondad de la gráfica en forma de ramificaciones, es que permite ver de manera clara, la forma en que se encuentran relacionadas las palabras dentro de cada cluster y con los demás cluster. En este caso, se puede observar en la Figura 4 la relación entre la palabra peligro (clusters2, con menor ocurrencia en comparación a las demás) con miedo (del cluster 3), de la misma que se relaciona por un lado con falta (del cluster 4), y por otro lado con robo (del cluster 1). Por lo tanto, se puede concluir que los hechos violentos aunados a la falta de control 
institucional (judicial), han permitido el establecimiento de un contexto reconstruido en términos de inseguridad-incertidumbre y que ha sido resignificado por los jóvenes desde un posicionamiento de miedo y peligrosidad.

\section{Discusión}

El estudio de la violencia a través de la TRS, ha permitido encontrar datos interesantes para su comprensión. El contenido de la representación que los jóvenes reconstruyen de la violencia, permite evidenciar que, desde su perspectiva, en Ecuador más que una violencia pragmática (teórica o cuantificada) existe un tipo de violencia menos visible que causa fuertes estragos en el tejido social y en la vida cotidiana de la sociedad (Palomeque, 2002; San Martín, 2013).

Los análisis de cluster arrojaron datos relevantes que evidencian dichos efectos y que se pueden categorizar en las tres dimensiones representacionales desde la perspectiva de Moscovici (1976). La primera del campo representacional, en el cual se encuentran las consecuencias que tiene la violencia en la vida cotidiana; en esta dimensión se encuentran los delitos como los robos o asaltos que los jóvenes pueden padecer en la vía pública. Esta dimensión obedece a la información general que se tiene de un fenómeno de interés y a través de las evidencias de este estudio, de la violencia (robos, asaltos y maltratos) que los jóvenes perciben en el contexto mediato (San Martin, 2013). La segunda dimensión informacional, hace referencia a las causas que han permitido el establecimiento y desarrollo de la violencia; los jóvenes denuncian la ineficiente estructura gubernamental como el principal problema que ha permitido el establecimiento de un contexto violento, ya que no existe un control de las instituciones judiciales lo que se traduce en una ausencia de vigilancia (San Martin, 2013). La tercera dimensión es la actitudinal, en la cual se destacan los efectos menos visibles de la violencia. Así, se enfatizan las emociones de miedo, incertidumbre o temor por el hecho de salir a la calle 
y llegar a ser víctima de algún tipo de delito (Cerrato \& Palmonari, 2007; Wagner et al., 2011).

La aproximación fenomenológica propuesta en esta investigación y en base a los resultados encontrados, puede ser una evidencia de que los jóvenes representan un tipo de violencia que se puede denominar cotidiana (Carrión, 2003; Molina et al., 2015; Palomeque, 2002). A los jóvenes les preocupa en mayor medida el delito y sus consecuencias intra e inter personales que se ven reflejadas en los sentimientos de temor, miedo o peligro. En este punto, se puede argumentar que las visiones teóricas de la violencia que se han establecido en Ecuador, no han aportado informaciones concretas en lo que concierne al contexto (Concha-Eastman, 2002), lo cual deja de lado aspectos que afectan a la sociedad; esto permite comprender que algunos factores que implica la violencia no se encuentran presentes en el discurso académico o político. De tal manera que, desde el discurso académico, se pueden encontrar categorizaciones de la violencia (Concha-Eastman, 2002) $\mathrm{y}$, desde las estadísticas gubernamentales, se encuentran los índices de delitos (INEC, 2011); sin embargo, ambos dejan de lado las consecuencias no tangibles de la violencia, como, por ejemplo, el temor, la incertidumbre, la desconfianza, etc. No obstante, los estudios sobre la violencia, desde las bases de datos, brindan información relevante acerca de los tipos de delitos que se cometen con más frecuencia. En efecto, los delitos más frecuentemente reportados, y consecuentemente contabilizados en bases de datos (robo o asalto), coinciden con los que existen en la RS de la violencia en los jóvenes de este estudio (Torres, 2005).

Las dos visiones de la violencia hasta aquí citadas, son de llamar la atención, ya que en Ecuador se han implementado mecanismo como los botones de pánico en los buses o taxis, con la finalidad de controlar o disminuir los hechos delictivos lo que, desde la perspectiva de las instituciones gubernamentales, reduciría el riesgo del delito. Sin embargo, estos no han contribuido a disminuir la percepción de inseguridad que tiene la población en general, como se pudo observar en el discurso de los jóvenes en el cual definen el contexto mediato como 
peligroso e inseguro. Estos resultados concuerdan con los encontrados por Molina-Coloma, Reyes-Sosa \& Larrañaga (2015); señalaron que, pese a las estrategias del gobierno, la percepción de inseguridad y delito se mantiene presente en el imaginario de la sociedad. De esta manera, se puede determinar que en el imaginario social existe un pensamiento colectivo de miedo e incertidumbre. Esto permite proponer que, a pesar de los esfuerzos gubernamentales hechos sobre la violencia, su dimensión simbólica no ha sido considerada en la estrategia gubernamental, por ende, encontramos una población temerosa e insegura.

En base a todo lo anterior, en la presente investigación, argumentamos que la visión de la violencia que ha emergido conjuntamente con el concepto de seguridad ciudadana, es una vía que permite aportar estrategias sobre la violencia (Palomeque, 2002). En la visión que tienen los jóvenes de este fenómeno se destacan las faltas que ellos atribuyen a las instituciones encargadas de reducir los índices delictivos, esto es equiparable a los factores institucionales como la impunidad o colusión. Por otra parte, desde la seguridad ciudadana se consideran factores situacionales que promueven la violencia en la vida cotidiana como, por ejemplo, el porte de armas o la vulnerabilidad que los sujetos tienen al salir a la calle o tomar un bus (Molina et al., 2015). Ciertamente, los jóvenes expresan su sentir de vulnerabilidad al dejar en claro las emociones negativas que ligan a la violencia. Menos visible en los resultados son los factores estructurales que tiene que ver con desigualdades sociales o precariedad social. En este sentido, promover estrategias para recuperar espacios de convivencia (por ejemplo, parques y universidades) puede permitir una reducción en la percepción de inseguridad y el delito; se debe recordar que la violencia reduce los espacios y los tiempos de convivencia entre la sociedad (MolinaColoma et al., 2015).

En suma, se puede concluir que el presente estudio ha permitido tener una comprensión más amplia de la violencia en Ecuador. De esta manera, si bien las perspectivas teóricas, de bases de datos y seguridad aportan elementos interesantes para comprender la violencia, también es cierto que han dejado de lado sus efectos entre la sociedad. En este 
sentido, la aproximación a la violencia desde la perspectiva de la TRS ha permitido evidenciar la importancia del aspecto subjetivo; como se ha observado en los resultados, desde esta perspectiva se puede evidenciar efectos menos visibles de objetos de relevancia e interés social. Además, desde una noción subjetiva se pueden evidenciar también los discursos $\mathrm{u}$ opiniones que demuestran las preocupaciones que los sujetos tienen frente un objeto en este caso la violencia (Rodríguez, 2003).

Por otra parte, el trabajo presentado no viene sin limitantes, el hecho de considerar solo a jóvenes universitarios restringe la comprensión del fenómeno de la violencia y sus efectos. Por último, el presente trabajo deja futuras líneas de investigación abiertas a exploración. Al seguir la idea de que diferentes grupos de personas construyen a distintas representaciones (Castillo, Evia \& Carrillo, 2017; Echeverría, 2017); en primera instancia, se sugiere un estudio en el cual el enfoque no sea únicamente en jóvenes universitarios, sino a una población más amplia que abarque otros grupos de edades, que, aunque sufren menos los efectos de la violencia (como delitos y robos) siguen siendo potenciales víctimas de ella. Además, estudios desde una perspectiva de género, que permitan identificar la RS y los efectos que tiene la violencia en base al grupo de pertenencia.

\section{Referencias}

Carneiro, J., Tsunoda, D. \& Giacomiti, R. (2017). Social media and self-medication for weight loss, insights from Facebook in Brazil. Journal of Engineering Research and Applications, 7(7), 26-36. https://doi.org/10.9790/9622-0707022636

Carrión, F. (2003). Ecuador, Seguridad Ciudadana y Violencia. Quito: Flacso-Ecuador.

Centro de Estudios y Datos (CEDATOS, 2011). La inseguridad en Ecuador. Recuperado de http://www.cedatos.com.ec/resultados. php?cx=009336540865533470268\%3Am7fwq4h04ts\&cof=FO RID\%3A10\&ie=UTF-8\&q=INSEGURIDAD\&sa.x=0\&sa. $y=0$ 
Cerrato, J. \& Palmonari, A. (2007). Representaciones sociales y psicología social. Comportamientos, globalización y posmodernidad. Promolibro: España.

Concha-Eastman, A. (2002). Salud, violencia e inseguridad. En F. Carrión (Ed.) Seguridad ciudadana, ¿espejismo o realidad? (pp. 503-520). Quito: Flacso-Ecuador, OPS/OMS.

Echeverría, R., Castillo, M. T., Evia, N. \& Carrillo, C. (2017). Representaciones sociales de líderes institucionales acerca del juego en casinos mexicanos. Revista de Psicología (PUCP), 35(1), 5-30. https://doi.org/10.18800/psico.201701.001

Farah, A. (2011). Attitude and social representation. Social and Behavioral Sciences, 30, 1593-1597. https://doi.org/10.1016/j.sbspro. 2011.10.309

Gaffié, B. (2012). Confrontations des Représentations Sociales et construction de la réalité. Journal International sur les Représentations Sociales, 2(1), 7-19. https://doi.org/10.4000/books. editionsehess. 1888

Garnier, B. \& Guérin-Pace, F. (2010). Appliquer les méthodes de la statistique textuelle. CEPED.

Greenacre, M. (2010). Correspondence analysis of raw data. Ecology, 91(4), 958-963. https://doi.org/10.1890/09-0239.1

Instituto Nacional de Estadística y Censos. (INEC, 2011). Encuesta de Victimización y Percepción de Inseguridad. Recuperado de: http:// www.ecuadorencifras.gob.ec/justicia-y-crimen/

Informe sobre Desarrollo Humano para América Central (IDHAC, 2010). Abrir espacios para la Seguridad Ciudadana y el Desarrollo Humano 2009-2010. Colombia: PNUD.

Markova, I. (2003). Dialogicality and Social Representations. The Dynamics of Mind. Cambridge: Cambridge University Press.

Molina-Coloma, V., Pérez, J. I. \& Salaberría, K. (2018). Diferencias generales y entre sexos en salud mental: Un estudio comparativo entre población penitenciaria y población general. Revista Mexicana de Psicología, 35(2), 117-130 
Molina-Coloma, V., Reyes-Sosa, H. \& Larrañaga-Egilegor, M. (2015). La representación social de la inseguridad en jóvenes universitarios ecuatorianos: el caso Ambato. Pensando Psicología, 11(8), 85-95. https://doi.org/10.16925/pe.v11i18.1221

Moscovici, S. (1976). La Psychanalyse: son Image et son Public. París: PUF.

Moscovici, S. (2001). Pourquoi l'étude des représentations sociales en psychologie? Psychologie et Société, 4(4), 7-27.

Moscovici, S. \& Duveen, G. (2000). Social Representation. Explorations in Social Psychology. Cambridge: Polity Press.

Núñez, J. (2011). Critica a la inseguridad ciudadana en Ecuador, 91 estrategias contra la violencia. Quito: Flacso.

Paéz, A. (2004). Hacia una propuesta de política pública de seguridad ciudadana. En O. Jarrin (ed.), Memoria del proyecto: politica pública de seguridad ciudadana primera fase (pp. 129-135). Quito: Flacso, Fundación ESQUEL.

Palomeque, E. (2002). Diagnóstico sobre seguridad ciudadana en el Ecuador. En F. Carrión (Ed.), Seguridad ciudadana, ¿espejismo o realidad? (pp. 235-249). Ecuador: Flacso.

Pontón, D. (2004). Políticas públicas en seguridad ciudadana: el caso Quito (2000-2004). En L. Dammert (ed.), Seguridad ciudadana experiencias y desafios (pp. 353-373. Chile: Ilustre Municipalidad de Valparaíso-Red 14.

Ramírez, F. (2011). Primera encuesta nacional sobre jóvenes y participación política en Ecuador. Recuperado de: http://www.activate.ec/ content/primera-encuesta-nacional-sobre-jovenes-y-participacion-politica-en-ecuador

Ratinaud, P. \& Déjean, S. (2009). IRaMuTeQ: implémentation de la méthode ALCESTE d'analyse de texte dans un logiciel libre. MASH: Modélisation Appliquée aux Sciences Humaines et sociales.

Rodríguez, T. (2003). El debate de las representaciones sociales en psicología social. Relaciones, 93(24), 53-80. 
San Martín, C. (2013). Las representaciones sociales de la seguridad ciudadana en los vecinos de la comuna de Melipilla, Chile. Psicoperspectivas, 12(1), 72-94. https://doi.org/10.5027/ psicoperspectivas-Vol12-Issue1-fulltext-219

Sarrica M. \& Contarello, A. (2004). Peace, war and conflict: Social representations shared by peace activists and non-activists. Journal of Peace Research, 41(5), 549-568. https://doi. org/10.1177/0022343304045976

Torres, A. (2005). La seguridad ciudadana en Ecuador un concepto en construcción: Estado del arte de investigaciones producidas entre los años 2000 y 2004. Quito: Flacso-Ecuador.

Valencia, J.-F., Gil-De-Montes, L. \& Elejabarrieta, F. (2004). Creencias y actitudes hacia la inmigración: estereotipos, prejuicio y regulaciones normativas. Revista de Psicología Social, 19(3), 299-318. https://doi.org/10.1174/0213474041960469

Wagner, W., Hayes, N. \& Flores, F. P. (2011). El discurso de lo cotidiano y el sentido común. La teoría de las Representaciones Sociales. Anthropos Editorial.

Recibido: 5 de setiembre, 2018

Revisado:31 de enero, 2019

Aceptado: 4 de febrero, 2019 